\title{
CHOW GROUPS, CHOW COHOMOLOGY, AND LINEAR VARIETIES
}

\author{
BURT TOTARO \\ UCLA Mathematics Department, Box 951555, Los Angeles, CA 90095-1555, USA; \\ email: totaro@math.ucla.edu
}

Received 21 October 2013; accepted 10 June 2014

\begin{abstract}
We compute the Chow groups and the Fulton-MacPherson operational Chow cohomology ring for a class of singular rational varieties including toric varieties. The computation is closely related to the weight filtration on the ordinary cohomology of these varieties. We use the computation to answer one of the open problems about operational Chow cohomology: it does not have a natural map to ordinary cohomology.
\end{abstract}

2010 Mathematics Subject Classification: 14C15 (primary); 14F42, 14M20 (secondary)

In 1995, Fulton, MacPherson, Sottile, and Sturmfels [13] succeeded in computing the Chow group $\mathrm{CH}_{*} \mathrm{X}$ of algebraic cycles and the 'operational' Chow cohomology ring $A^{*} X$ [12] for a class of singular algebraic varieties. The varieties they consider are those which admit a solvable group action with finitely many orbits; this includes toric varieties and Schubert varieties. In this paper, we generalize their theorem that $A^{i} X \cong \operatorname{Hom}\left(C H_{i} X, \mathbf{Z}\right)$ to the broader class of linear schemes $X$, as defined below. We compute explicitly the Chow groups and the weight-graded pieces of the rational homology of those linear schemes which are finite disjoint unions of pieces isomorphic to $\left(G_{m}\right)^{a} \times A^{b}$ for some $a, b$. We show that the Chow groups $\otimes \mathbf{Q}$ of any linear scheme map isomorphically to the lowest subspace in the weight filtration of rational homology. Finally, we find some special properties of toric varieties (splitting of the weight filtration on their rational homology and existence of a map $A^{i} X \otimes \mathbf{Q} \rightarrow H^{2 i}(X, \mathbf{Q})$ with good

(c) The Author 2014. The online version of this article is published within an Open Access environment subject to the conditions of the Creative Commons Attribution licence $<$ http://creativecommons.org/licenses/by/3.0/>. 
properties) which do not extend to arbitrary linear schemes, as is shown by an interesting example (a surface with a cusp singularity). We formulate some open problems about Chow cohomology in Section 8.

We define the class of linear schemes to consist of the schemes over a fixed field $k$ which can be obtained by an inductive procedure starting with affine space of any dimension, in such a way that the complement of a linear scheme imbedded in affine space is also a linear scheme, and a scheme which can be stratified as a finite disjoint union of linear schemes is a linear schemes. (This class of schemes is a slight variant of a class of schemes studied by Jannsen [16]; see Section 2.)

Every scheme which admits an action of a split solvable group with finitely many orbits is a linear scheme, since each orbit is isomorphic to $\left(G_{m}\right)^{a} \times A^{b}$ for some $a, b$. Some examples of linear schemes which do not have a solvable group action with finitely many orbits are the intersection of two Schubert varieties (studied by Deodhar and others in connection with the Kazhdan-Lusztig polynomials), the discriminant hypersurface $\{\Delta=0\} \subset \mathbf{C}^{n}$, and at least some quotients of affine space by finite groups (these have interesting torsion in their Chow groups).

Here are the main theorems.

THEOREM 1. We give explicit generators and relations for the Chow groups of any scheme over a field $k$ which can be stratified into finitely many pieces isomorphic to $\left(G_{m}\right)^{a} \times A^{b}$.

In particular, Theorem 1 applies to varieties on which a split solvable group acts with finitely many orbits. (See the end of Section 2.)

THEOREM 2. For any linear variety $X$ which is proper over $k$, the FultonMacPherson operational Chow ring $A^{*} X[12]$ has

$$
A^{i} X \cong \operatorname{Hom}\left(C H_{i} X, \mathbf{Z}\right)
$$

For a linear scheme $X$ over the complex numbers, we can relate the Chow groups to the weight filtration on the ordinary homology of $X$ (Borel-Moore homology if $X$ is noncompact). (See [6] and [15] for the weight filtration on Borel-Moore homology. They actually discuss the mixed Hodge structure on cohomology with compact support, which is equivalent since $H_{i}^{B M}(X, \mathbf{Q})$ is dual to $H_{c}^{i}(X, \mathbf{Q})$ for any complex scheme $X$. For any complex scheme, the weight filtration of $H_{k}^{B M}(X, \mathbf{Q})$ is an increasing filtration with associated graded groups in degrees $-k$ to 0 .) 
THEOREM 3. For any linear scheme $X$ over the complex numbers, the natural map

$$
C H_{i} X \otimes \mathbf{Q} \rightarrow W_{-2 i} H_{2 i}^{B M}(X, \mathbf{Q})
$$

from the Chow groups into the smallest subspace of Borel-Moore homology with respect to the weight filtration is an isomorphism.

The surjectivity of this map was proved by Jannsen [16].

THEOREM 4. For any scheme over the complex numbers which is stratified as a finite disjoint union of varieties isomorphic to products $\left(G_{m}\right)^{a} \times A^{b}$, we give an explicit chain complex whose homology computes the weight-graded pieces of the Borel-Moore homology $H_{*}^{B M}(X, \mathbf{Q})$.

THEOREM 5. For each toric variety $X$, there is a natural grading of each group $H_{i}^{B M}(X, \mathbf{Q})$ and $H^{i}(X, \mathbf{Q})$ which splits the weight filtration and is compatible with products and mixed Hodge structures.

THEOREM 6. If $X$ is a compact toric variety over the complex numbers, then there is a natural isomorphism

$$
A^{i} X \otimes \mathbf{Q} \stackrel{\cong}{\longrightarrow} H^{2 i}(X, \mathbf{Q}) \cap F^{i} H^{2 i}(X, \mathbf{C}) .
$$

THEOREM 7. There is no functorial homomorphism $A^{1} X \otimes \mathbf{Q} \rightarrow H^{2}(X, \mathbf{Q})$ for general complex varieties $X$, or even for normal projective linear varieties, which agrees with the usual homomorphism for smooth $X$ and which is well behaved in families (see Section 7 for details).

As background for Theorems 6 and 7 we have the following. It was an open problem for a long time to decide whether there was a natural map $A^{i} X \rightarrow$ $H^{2 i}(X, \mathbf{Z})$ for all complex varieties $X$. This is true for smooth varieties, since $A^{i} X$ coincides with the Chow group of codimension- $i$ cycles on $X$ in that case. In 1995, Bloch, Gillet, and Soule [5] showed that there is a natural map from $A^{i} X \otimes \mathbf{Q}$ to $\operatorname{gr}_{W}^{2 i} H^{2 i}(X, \mathbf{Q})$, which is a quotient of $H^{2 i}(X, \mathbf{Q})$ for $X$ compact. In fact it maps into Hodge filtration $F^{i}$ of this quotient. For compact toric varieties, Theorem 6 lifts this map into $H^{2 i}(X, \mathbf{Q}) \cap F^{i} H^{2 i}(X, \mathbf{C})$; but Section 7 gives an example of a linear variety $Y$ (a surface with a single cusp singularity) for which the map does not so lift. By analyzing a family of varieties containing $Y$, we can see that there is no map $A^{i} X \otimes \mathbf{Q} \rightarrow H^{2 i}(X, \mathbf{Q})$ with good properties in general.

An earlier version of this paper was written around 1996. A number of papers over the past 15 years have referred to it, and so it seems reasonable to make it 
permanently available. Following a referee's suggestion, I added some comments on the relation between higher Chow groups and Voevodsky's motivic homology groups. I also added some papers to the bibliography which refer to the preprint version of this paper, and which develop the ideas further $[7,10,14,17,23]$.

\section{Examples of toric varieties}

In this section, we recall some examples of singular compact toric varieties from Fulton [11], showing the basic phenomena which inspired some of the general theorems in the rest of the paper: the Chow groups can have torsion, and the homology can be bigger than the Chow groups, even rationally. These things do not happen for smooth compact toric varieties.

A toric variety is described by a fan, which is an arrangement of rational polyhedral cones in $N \otimes \mathbf{R}$ for a lattice $N \cong \mathbf{Z}^{n}$ such that a face of a cone in the fan is a cone in the fan, and the intersection of two cones is a face of each. Let $X$ be the compact toric surface corresponding to the fan in $\mathbf{Z}^{2}$ with edges through the points $(2,-1),(-1,2)$, and $(-1,-1)$. Then

$$
C H_{1} X=\mathbf{Z} \oplus \mathbf{Z} / 3,
$$

as a result of the three points $(2,-1),(-1,2)$, and $(-1,-1)$ all being equal in $N \otimes \mathbf{Z} / 3$ [11, p. 65]. Similar examples give toric surfaces with $\mathbf{Z} / n$-torsion in $C H_{1} X$ for any $n$. For compact toric surfaces, $C H_{*} X$ maps isomorphically to $H_{*}(X, \mathbf{Z})$.

Next, consider the fan with edges through the vertices $( \pm 1, \pm 1, \pm 1)$ of a cube, in the sublattice of $\mathbf{Z}^{3}$ generated by these vertices [11, p. 105]. Then the corresponding compact toric threefold has $\mathrm{CH}_{0} \mathrm{X}=\mathrm{H}_{0}(\mathrm{X}, \mathbf{Z})=\mathbf{Z}, \mathrm{CH}_{3} \mathrm{X}=$ $H_{0}(X, \mathbf{Z})=\mathbf{Z}$, and

$$
C H_{1} X=H_{2} X=\mathbf{Z}, \quad H_{3} X=\mathbf{Z}^{2}, \quad C H_{2} X=H^{4} X=\mathbf{Z}^{5} .
$$

Other examples (for a trivial one, take the product of this variety with itself) show that even the even-dimensional homology can be bigger than the Chow groups.

\section{Proof of Theorem 1}

Proof. We use Bloch's higher Chow groups [3]. Levine showed that these groups satisfy a localization exact sequence for all separated schemes of finite type over a field, generalizing Bloch's proof in the quasi-projective case; see [4] and [21, Theorem 0.7].

Let us note the comparison between higher Chow groups and Voevodsky's (Borel-Moore) motivic homology groups, although it will not be needed in 
this paper. Voevodsky's motivic homology groups were initially defined when the base field $k$ admits resolution of singularities [22, Definition 16.20]. More recently, Kelly generalized Voevodsky's definition to any field, at the cost of considering only motivic homology with a coefficient ring in which the exponential characteristic of the field is invertible [18, Proposition 5.5.5]. These motivic homology groups agree with Bloch's higher Chow groups for separated schemes of finite type over a field, under the same restriction on coefficients, by the arguments of [21, Theorem 0.7], [22, Proposition 19.18], and [27, Corollary 2].

Note that Bloch's higher Chow groups $C H^{i}(X, j)$ have the functorial properties of a (Borel-Moore) homology theory, despite being written with a superscript. For example, for a singular scheme $X$, the groups $C H^{*}(X, 0)$ are the usual Chow groups of $X$ (indexed so that $\left.C H_{i}(X)=C H^{\operatorname{dim} X-i}(X, 0)\right)$. As a result, the notation $C H^{i}(X, j)$ is only reasonable when $X$ is equidimensional. In general, for an equidimensional scheme $X$ over $k$, we define motivic homology as

$$
H_{a}^{M}(X, \mathbf{Z}(b)):=C H^{\operatorname{dim} X-b}(X, a-2 b) .
$$

If $X$ is not equidimensional, then $H_{a}^{M}(X, \mathbf{Z}(b))$ makes sense from Bloch's definition, whereas $C H^{i}(X, j)$ does not. On the other hand, it always makes sense to write

$$
C H^{*}(X, j):=\oplus_{b} H_{2 b+j}^{M}(X, \mathbf{Z}(b)) .
$$

All the tori we mention will be assumed to be split, that is, isomorphic to $\left(G_{m}\right)^{a}$ over some base scheme. Also, it is important to understand the meaning of the word 'stratified' in the statement of the theorem: this means that the scheme $X$ we are given is a finite disjoint union of locally closed subvarieties, here assumed to be isomorphic to $\left(G_{m}\right)^{a} \times A^{b}$ for various $a, b$, such that the closure of an $i$-dimensional piece $S$, minus $S$, is contained in the union of the pieces of dimension less than $i$.

For any scheme $X$ over $k$, the motivic homology of $G_{m} \times_{k} X$ is given by

$$
H_{a}^{M}\left(G_{m} \times X, \mathbf{Z}(b)\right)=H_{a-2}^{M}(X, \mathbf{Z}(b-1)) \oplus H_{a-1}^{M}(X, \mathbf{Z}(b)) .
$$

This follows from homotopy invariance of the motivic homology groups together with the localization sequence

$$
\cdots \rightarrow H_{2 i+1}^{M}(U, \mathbf{Z}(i)) \rightarrow H_{2 i}^{M}(S, \mathbf{Z}(i)) \rightarrow H_{2 i}^{M}(X, \mathbf{Z}(i)) \rightarrow H_{2 i}^{M}(U, \mathbf{Z}(i)) \rightarrow 0
$$

for $S$ a closed subscheme of $X$ with complement $U$. The last three groups in this exact sequence are just the usual Chow groups of $S, X$, and $U$. 
In the notation $C H^{*}(X, j)$ discussed above, we have

$$
C H^{*}\left(G_{m} \times_{\mathbf{Z}} X, *\right)=C H^{*}(X, *) \bigotimes_{\mathbf{Z}} \Lambda^{*} M,
$$

where $M \cong \mathbf{Z}$ is in degree $(1,1)$. Using this formula repeatedly, we can compute the motivic homology of a general split torus $T$ :

$$
C H^{*}\left(T \times_{\mathbf{Z}} X, *\right)=C H^{*}(X, *) \bigotimes_{\mathbf{Z}} \Lambda^{*} M,
$$

where the finitely generated free abelian group $M=\operatorname{Hom}\left(T, G_{m}\right)$ is in degree $(1,1)$.

In the standard notation for motivic homology, this formula looks a little more complicated: if $T$ is a $d$-dimensional torus over a field $k$, then

$$
H_{a}^{M}(T, \mathbf{Z}(b))=\oplus_{j} \Lambda^{j} M \bigotimes_{\mathbf{Z}} H_{a-2 d+j}^{M}(k, \mathbf{Z}(b-d+j)) .
$$

Moreover, the same formula holds if $T$ is a $d$-dimensional variety which is a product $T_{0} \times A^{d-r}$, where $T_{0}$ is a torus of dimension $r$, and we define $M=$ $\operatorname{Hom}\left(T_{0}, G_{m}\right)$. This follows from the homotopy invariance of motivic homology.

Now we can begin our analysis of the usual Chow groups of a $k$-scheme cut into pieces $T=\left(G_{m}\right)^{a} \times A^{b}$. Write $M(T)$ for the finitely generated free abelian group $\operatorname{Hom}\left(\left(G_{m}\right)^{a}, G_{m}\right)$. Since we have a stratification of the scheme $X$, the union $X_{i}$ of the pieces of dimension $\leqslant i$ is closed (for all $i$ ), and the difference $X_{i}-X_{i-1}$ is isomorphic to the disjoint union of the $i$-dimensional pieces $T_{i}$. From the localization sequence for motivic homology, we get a spectral sequence converging to the motivic homology of $X$ of weight $i$,

$$
E_{p q}^{1}=H_{p+q}^{M}\left(T_{p}, \mathbf{Z}(i)\right) \Rightarrow H_{p+q}^{M}(X, \mathbf{Z}(i)) .
$$

This spectral sequence has homological numbering, meaning that the differential $d_{i}$ has bidegree $(-i, i-1)$.

$$
\begin{array}{r}
\oplus H_{2 i}^{M}\left(T_{d-2}, \mathbf{Z}(i)\right) \longleftarrow \oplus H_{2 i+1}^{M}\left(T_{d-1}, \mathbf{Z}(i)\right) \longleftarrow \oplus H_{2 i+2}^{M}\left(T_{d}, \mathbf{Z}(i)\right) \\
\oplus H_{2 i}^{M}\left(T_{d-1}, \mathbf{Z}(i)\right) \longleftarrow \oplus H_{2 i+1}^{M}\left(T_{d}, \mathbf{Z}(i)\right) \\
\oplus H_{2 i}^{M}\left(T_{d}, \mathbf{Z}(i)\right)
\end{array}
$$

The bottom diagonal (NW to SE) corresponds to the usual Chow groups $C H_{i} X=H_{2 i}^{M}(X, \mathbf{Z}(i))$, the diagonal above that to $H_{2 i+1}^{M}(X, \mathbf{Z}(i))$, and so on. The direct sums shown run over the sets of pieces $T_{r}$ of a given dimension $r$. 
We now write this spectral sequence in more detail, using the computation of the motivic homology of the pieces $T_{i} \cong\left(G_{m}\right)^{a} \times A^{i-a}$. The computation is expressed in terms of the motivic homology of the base field $k$, and so I will first summarize some of the simpler results about these groups. First,

$$
H_{a}^{M}(k, \mathbf{Z}(b))=0 \text { if } a<2 b \text { or if } a<b
$$

by the definition of higher Chow groups. Next,

$$
\begin{aligned}
& H_{2 b}^{M}(k, \mathbf{Z}(b))= \begin{cases}\mathbf{Z} & \text { if } b=0 \\
0 & \text { otherwise }\end{cases} \\
& H_{2 b+1}^{M}(k, \mathbf{Z}(b))= \begin{cases}k^{*} & \text { if } b=-1 \\
0 & \text { otherwise. }\end{cases}
\end{aligned}
$$

These facts are all we need in order to compute the classical Chow groups $C H_{i}(X)=H_{2 i}^{M}(X, \mathbf{Z}(i))$ for the scheme $X$ which can be cut into pieces $\left(G_{m}\right)^{a} \times A^{b}$. Namely, they imply that $H_{a}^{M}\left(T_{r}, \mathbf{Z}(i)\right)$ is nonzero only if $a \geqslant r+i$ and $a \geqslant 2 i$; that is, the $E_{1}$ term of the spectral sequence is concentrated in rows $\geqslant i$, and on or above the diagonal corresponding to $H_{2 i}^{M}(X, \mathbf{Z}(i))=C H_{i} X$. Moreover, the only nonzero group on this diagonal is $\oplus_{T_{i}} H_{2 i}^{M}\left(T_{i}, \mathbf{Z}(i)\right)=\oplus_{T_{i}} \mathbf{Z}$. It follows that the spectral sequence degenerates at $E_{2}$ on this diagonal, so that

$$
\begin{aligned}
C H_{i} X & =\oplus_{T_{i}} H_{2 i}^{M}\left(T_{i}, \mathbf{Z}(i)\right) / \oplus_{T_{i+1}} H_{2 i+1}^{M}\left(T_{i+1}, \mathbf{Z}(i)\right) \\
& =\oplus_{T_{i}} \mathbf{Z} / \oplus_{T_{i+1}}\left(M\left(T_{i+1}\right) \oplus k^{*}\right) .
\end{aligned}
$$

To clarify this, we draw a picture of the spectral sequence below, using a few additional facts about the motivic homology of $k: H_{a}^{M}(k, \mathbf{Z}(0))$ is $\mathbf{Z}$ if $a=0$ and zero otherwise; $H_{a}^{M}(k, \mathbf{Z}(-1))$ is $k^{*}$ if $a=-1$ and zero otherwise [3, Theorem 6.1]; $H_{-2}^{M}(k, \mathbf{Z}(-2))=K_{2} k, H_{-3}(k, \mathbf{Z}(-3))$ is the Milnor $K$-group $K_{3}^{M} k$, and $H_{-1}^{M}(k, \mathbf{Z}(-2))$ is $K_{3}^{\text {ind }} k:=K_{3}(k) / K_{3}^{M}(k)$. The bottom diagonal gives the Chow group $C H_{i} X$ :

$$
\begin{array}{ccc}
0 & 0 & \oplus_{T_{i+2}} K_{3}^{\text {ind }} k \\
& \oplus_{T_{i}} \mathbf{Z} \longleftarrow \oplus_{T_{i+1}}\left(M\left(T_{i+1}\right) \oplus k^{*}\right) \longleftarrow \oplus_{T_{i+2}}\left(\Lambda^{2} M\left(T_{i+2}\right) \oplus\left(M\left(T_{i+2}\right) \otimes_{\mathbf{Z}} k^{*}\right) \oplus K_{2} k\right)
\end{array}
$$

Clearly we can say more about this spectral sequence than just its degeneration on the diagonal corresponding to the Chow groups; but we put this aside for now. 
Let us just mention that the $d_{1}$ differential $\oplus_{T_{i+1}}\left(M\left(T_{i+1}\right) \oplus k^{*}\right) \rightarrow \oplus_{T_{i}} \mathbf{Z}$ is 0 on each copy of $k^{*}$, so that we can describe $C H_{i} X$ just as $\oplus_{T_{i}} \mathbf{Z} / \oplus_{T_{i+1}} M\left(T_{i+1}\right)$. This is a special case of the following lemma. Theorem 1 is proved.

LEMMA 1. Let $S$ be a closed subscheme of a scheme $X$ over $k$, let $U=X-S$, and let $Y$ be another $k$-scheme. Then the composition of the following product map and boundary map is 0 :

$$
C H^{*} U \bigotimes_{\mathbf{Z}} C H^{*}(Y, 1) \rightarrow C H^{*}(U \times Y, 1) \rightarrow C H^{*}(S \times Y)
$$

Proof. We have a surjection $C H^{*} X \rightarrow C H^{*} U$, which implies that $C H^{*} X \bigotimes_{\mathrm{z}} C H^{*}(Y, 1) \rightarrow C H^{*} U \bigotimes_{\mathrm{Z}} C H^{*}(Y, 1)$ is surjective. So it suffices to show that the map from $C H^{*} X \bigotimes_{\mathrm{z}} C H^{*}(Y, 1)$ to $C H^{*}(S \times Y)$ is 0 . But this map is just the composition of the product $C H^{*} X \bigotimes_{\mathbf{Z}} C H^{*}(Y, 1) \rightarrow C H^{*}(X \times Y, 1)$ with the composition

$$
C H^{*}(X \times Y, 1) \rightarrow C H^{*}(U \times Y, 1) \rightarrow C H^{*}(S \times Y),
$$

and this last composition is 0 (it is part of the localization sequence).

Addendum. Rosenlicht showed that a homogeneous space for a split solvable group over a field $k$ is isomorphic as a variety to $\left(G_{m}\right)^{a} \times A^{b}$ for some $a, b$ [24, p. 119]. (A solvable group is said to be split over $k$ if it is a successive extension of the additive and multiplicative groups over $k, G_{a}$ and $G_{m}$. Every smooth connected solvable group over an algebraically closed field is split.)

It follows that the varieties considered by Fulton, MacPherson, Sottile, and Sturmfels [13], that is, varieties over an algebraically closed field which admit a solvable group action with finitely many orbits, are included among those considered in Theorem 1. In particular, such varieties are linear schemes in the sense defined below.

\section{Proof of Theorem 2 and the Chow Künneth property for linear schemes}

We begin by defining the class of varieties considered in the theorem. The class of linear schemes over a field $k$ is the class of schemes obtained by an inductive procedure starting with affine space of any dimension over $k$, in such a way that the complement of a linear scheme imbedded in affine space (in any way) is a linear scheme, and a scheme stratified (cf. Section 2) into a finite disjoint union of linear schemes is a linear schemes. The property of being a linear scheme only depends on the underlying reduced scheme. This definition is inspired by 
Jannsen's definition of a slightly broader class of schemes, which he called linear varieties [16]. (In Jannsen's similar inductive procedure, the complement of a linear variety imbedded in any linear variety, not just in affine space, is called a linear variety. In this paper, I will always use the phrase 'linear scheme' in the narrower sense defined above.)

Fortunately, all the examples of Jannsen's class of varieties which he mentions do belong to this paper's class of linear schemes, so it seems silly to worry about the difference too much. The examples include complements in $\mathbf{P}^{n}$ (or $A^{n}$ ) of a union of linear subspaces, successive blow-ups of $\mathbf{P}^{n}$ in linear subspaces, and schemes stratified by such varieties, such as Schubert varieties and toric varieties. Our interest is in singular schemes, but of course such smooth varieties as Grassmannians and flag varieties are included.

We will deduce Theorem 2 , that $A^{i} X \cong \operatorname{Hom}\left(\mathrm{CH}_{i} X, \mathbf{Z}\right)$ for proper linear varieties $X$ over $k$, from the following two propositions.

PROPOSITION 1. If $X$ is a linear scheme and $Y$ is any scheme of finite type over $k$, then

$$
C H_{*} X \bigotimes_{\mathbf{Z}} C H_{*} Y \stackrel{\cong}{\rightarrow} C H_{*}(X \times Y) .
$$

PROPOSITION 2. If $X$ is a proper $k$-variety such that

$$
C H_{*} X \bigotimes_{\mathbf{Z}} C H_{*} Y \stackrel{\cong}{\rightarrow} C H_{*}(X \times Y)
$$

for all schemes $Y$ of finite type over $k$, then the cap product maps $A^{i} X \rightarrow$ $\operatorname{Hom}\left(\mathrm{CH}_{i} \mathrm{X}, \mathbf{Z}\right)$ are isomorphisms.

We only outline the proof of Proposition 2, which is one of the main results of Fulton, MacPherson, Sottile, and Sturmfels [13]. By definition, an element $c \in$ $A^{i} X$ is a collection of homomorphisms $C H_{m} Y \rightarrow C H_{m-i} Y$, written $z \mapsto f^{*} c \cap z$, for all maps $f: Y \rightarrow X$ and all integers $m$, which satisfy three compatibility conditions: with proper pushforward, flat pullback, and intersection with a divisor, whenever one has maps $Y^{\prime} \rightarrow Y \rightarrow X$ with $Y^{\prime} \rightarrow Y$ proper, or flat, or the inclusion of a Cartier divisor. The main step in the proof of Proposition 2 is, given an abelian group homomorphism $\phi: C H_{i} X \rightarrow \mathbf{Z}$, to construct an element $C_{\phi} \in A^{i} X$, assuming that $X$ satisfies the Chow Künneth property. Thus, for every map $f: Y \rightarrow X$ and every $m$, we have to construct a homomorphism from $C H_{m} Y$ to $C H_{m-i} Y$. This homomorphism is defined to be the composite 


$$
\begin{aligned}
C H_{m} Y & \rightarrow C H_{m}(X \times Y)=\oplus_{j}\left(C H_{j} X \otimes C H_{m-j} Y\right) \\
& \rightarrow C H_{i} X \otimes C H_{m-i} Y \rightarrow \mathbf{Z} \otimes C H_{m-i} Y=C H_{m-i} Y .
\end{aligned}
$$

The maps here are clear except for the first one, which is induced by the inclusion of $Y$ into $X \times Y$ via the graph of $f: Y \rightarrow X$. One checks that these maps (for different schemes $Y$ ) satisfy the compatibility conditions to give an element of $A^{i} Y$, and that every element of $A^{i} X$ is so obtained. (We need properness of $X$ for this last step, in order to have a degree map $\mathrm{CH}_{0} X \rightarrow \mathbf{Z}$.)

We now turn to the proof of Proposition 1.

The higher Chow groups $C H^{i}(X, *)$ are defined as the homology of a complex $z^{i}(X, *)$ of free abelian groups [3]. It will be convenient to consider, for varieties $X$ and $Y$, the homology of the complex $z^{i}(X, *) \bigotimes_{\mathrm{Z}} z^{j}(Y, *)$; call it $C H^{i, j}(X, Y$, $*)$. By the Künneth formula for chain complexes, we have

$$
C H^{i, j}(X, Y, 0)=C H^{i} X \bigotimes_{\mathbf{Z}} C H^{j} Y,
$$

whereas for $C H^{i, j}(X, Y, 1)$ there is only an exact sequence

$$
\begin{aligned}
0 & \rightarrow\left(C H^{i} X \bigotimes_{\mathbf{Z}} C H^{j}(Y, 1)\right) \oplus\left(C H^{i}(X, 1) \bigotimes_{\mathbf{Z}} C H^{j} Y\right) \rightarrow C H^{i, j}(X, Y, 1) \\
& \rightarrow \operatorname{Tor}_{1}^{\mathbf{Z}}\left(C H^{i} X, C H^{j} Y\right) \rightarrow 0 .
\end{aligned}
$$

The point is that the product on the higher Chow groups is given by a map from a subcomplex of $z^{i}(X, *) \bigotimes_{\mathbf{z}} z^{j}(Y, *)$ with the same homology to the complex $z^{i+j}(X \times Y, *)$; so the product gives a map

$$
C H^{i, j}(X, Y, *) \rightarrow C H^{i+j}(X \times Y, *) .
$$

Consider the following two properties of schemes.

Weak property. A scheme $X$ of finite type over $k$ has the weak property (or: 'satisfies the Chow Künneth property') if the natural map

$$
C H^{*} X \bigotimes_{\mathbf{Z}} C H^{*} Y \rightarrow C H^{*}(X \times Y)
$$

is an isomorphism for all schemes $Y$ of finite type over $k$. (Be warned that we are writing $C H^{*} X$ for the direct sum $\oplus_{j} C H_{j} X$.)

Strong property. A scheme $U$ of finite type over $k$ has the strong property if it satisfies the weak property and the map

$$
\oplus C H^{*, *}(U, Y, 1) \rightarrow C H^{*}(U \times Y, 1)
$$

is surjective for all schemes $Y$ of finite type over $k$. 
We will prove by induction on dimension that the complement of any linear scheme imbedded in affine space satisfies the strong property and, simultaneously, that any quasi-projective linear scheme satisfies the weak property. The latter statement is the theorem we are trying to prove.

LEMMA 2. Affine space $A^{n}$ satisfies the strong property.

Proof. Use homotopy invariance of the higher Chow groups.

LEMMA 3. Let $S$ be a closed subscheme of a separated scheme $X$ of finite type over $k$, and let $U=X-S$. If $X$ satisfies the strong property and $S$ the weak, then $U$ satisfies the strong property.

Proof. We have the localization sequence

$$
C H^{*}(X, 1) \rightarrow C H^{*}(U, 1) \rightarrow C H^{*}(S, 0) \rightarrow C H^{*}(X, 0) \rightarrow C H^{*}(U, 0) \rightarrow 0
$$

which comes from an exact triangle of complexes of free abelian groups, $z^{*}(S$, $*) \rightarrow z^{*}(X, *) \rightarrow z^{*}(U, *)$. (As noted in Section 2, the total group $C H^{*}(X, j)$ makes sense even if $X$ is not equidimensional.) Therefore we can tensor this exact triangle over $\mathbf{Z}$ with any complex of abelian groups, in particular with $z^{*}(Y, *)$ for a scheme $Y$ over $k$, and we get another exact triangle. Taking homology gives a long exact sequence, with product maps as shown:

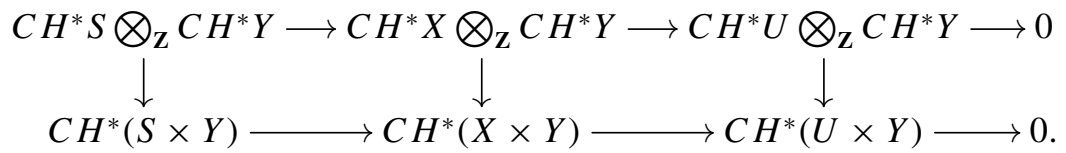

The first and second vertical arrows are isomorphisms, and so the third is also an isomorphism. Continuing these sequences to the left, we have

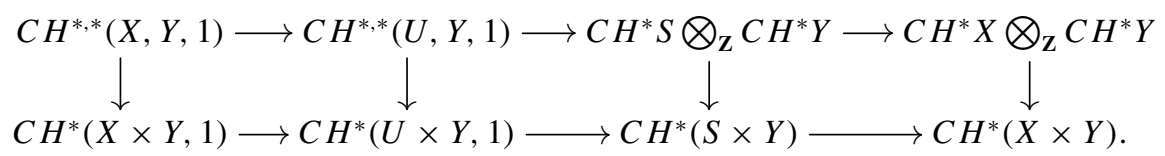

The first vertical arrow is surjective, and the third and fourth vertical arrows are isomorphisms. Diagram chasing shows that the second map is surjective.

LEMMA 4. Let $S$ be a closed subscheme of a separated scheme $X$ of finite type over $k$, and let $U=X-S$. If $U$ satisfies the strong property and $S$ the weak, then $X$ satisfies the weak property. 
Proof. As in the previous lemma, we have a map of long exact sequences:

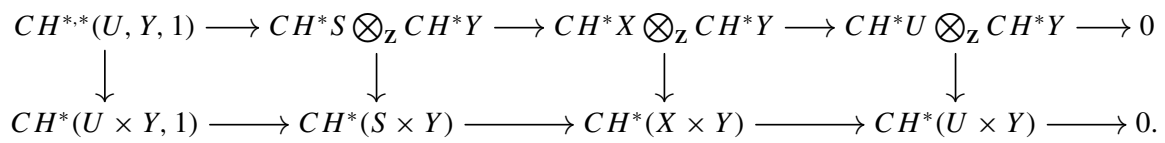

This time, the first vertical map is surjective, and the second and the fourth are isomorphisms. Diagram chasing shows that the third map is an isomorphism.

That completes the proof of Proposition 1. Together with Proposition 2, this implies Theorem 2.

\section{Chow groups and ordinary homology (proof of Theorem 3)}

For a scheme $X$ over a field $k$, we can try to construct homomorphisms from the motivic homology groups $H_{a}^{M}(X, \mathbf{Z}(b))$ to any reasonable homology theory for algebraic varieties. This is possible at least after tensoring motivic homology groups with $\mathbf{Q}$, because they agree $\otimes \mathbf{Q}$ with the Adams-graded pieces of the $K$ theory $G_{*} X$ of coherent sheaves on $X$, from which Gillet has defined maps into all reasonable homology theories. In particular, for $X$ defined over a subfield $k \subset \mathbf{C}$, there is a natural map

$$
H_{a}^{M}(X, \mathbf{Q}(b)) \rightarrow W_{-2 b} H_{a}^{B M}(X, \mathbf{Q}) \cap F^{-b} H_{a}^{B M}(X, \mathbf{C}) .
$$

(See Jannsen [16, 8.4.3 and 8.8, pp. 127-128].) Moreover, the resulting map $H_{a}^{M}(X, \mathbf{Q}(b)) \rightarrow H_{a}^{B M}(X, \mathbf{Q})$ is compatible with the usual long exact sequences.

We will only use that there is a map from $H_{a}^{M}(X, \mathbf{Q}(b))$ to $W_{-2 b} H_{a}^{B M}(X, \mathbf{Q})$, and therefore to $W_{-2 b} / W_{-2 b-1}=\mathrm{gr}_{-2 b}^{W}$, which is compatible with long exact sequences. For example, when $a=2 b$, this says that the usual Chow groups of $X$, tensored with $\mathbf{Q}$, map into the smallest weight subspace of the Borel-Moore homology of $X$.

Consider the following two properties of schemes of finite type over a subfield $k \subset \mathbf{C}$.

Weak property. A scheme $X$ over $k$ satisfies the weak property if the natural map

$$
H_{2 i}^{M}(X, \mathbf{Q}(i))=C H_{i} X \otimes \mathbf{Q} \rightarrow W_{-2 i} H_{2 i}^{B M}(X, \mathbf{Q})
$$

is an isomorphism for all $i$.

Strong property. A scheme $X$ over $k$ satisfies the strong property if it satisfies the weak property and, in addition, the map

$$
H_{2 i+1}^{M}(X, \mathbf{Q}(i)) \rightarrow \operatorname{gr}_{-2 i}^{W} H_{2 i+1}^{B M}(X, \mathbf{Q})
$$

is surjective. 
We will prove by induction on dimension that the complement of any linear scheme over $k$ imbedded in affine space satisfies the strong property and, simultaneously, that any linear scheme satisfies the weak property. The latter statement is the theorem we are trying to prove. This is exactly parallel to the proof of the Chow Künneth property for linear schemes in Section 3.

LEMMA 5. Affine space $A^{n}$ over $k$ satisfies the strong property.

Proof. $C H_{n} A^{n} \otimes \mathbf{Q}=\mathbf{Q}$ maps isomorphically to $W_{-2 n} H_{2 n}^{B M}\left(A^{n}, \mathbf{Q}\right)$, and all the rest of the Borel-Moore homology of $A^{n}$ is 0 .

LEMMA 6. Let $S$ be a closed subscheme of a separated scheme $X$ of finite type over $k$, and let $U=X-S$. If $X$ satisfies the strong property and $S$ the weak, then $U$ satisfies the strong property.

Proof. We have a map of localization sequences:

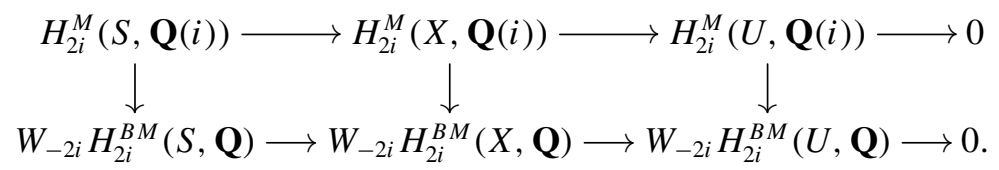

The first and second vertical arrows are isomorphisms, and so the third is also an isomorphism. Looking further to the left in these sequences, we have

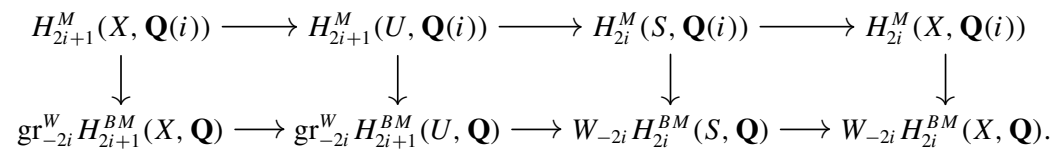

The first vertical arrow is surjective, and the third and fourth vertical arrows are isomorphisms. Diagram chasing shows that the second map is surjective.

LEMMA 7. Let $S$ be a closed subscheme of a separated scheme $X$ of finite type over $k$, and let $U=X-S$. If $U$ satisfies the strong property and $S$ the weak, then $X$ satisfies the weak property.

Proof. As in the previous lemma, we have a map of long exact sequences

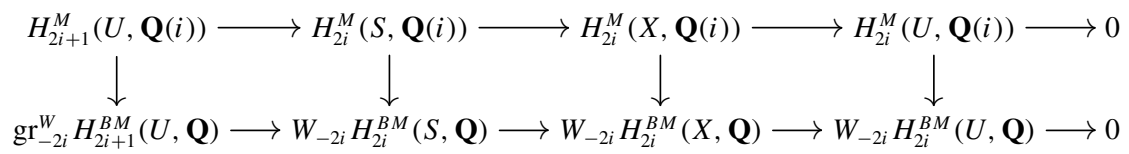

in which the first vertical map is surjective, and the second and the fourth are isomorphisms. Diagram chasing shows that the third map is an isomorphism. 
The lemmas imply that any linear scheme over $k$ satisfies the weak property. That is, for any linear scheme, the Chow groups inject into ordinary homology after tensoring with $\mathbf{Q}$, and the image is exactly the smallest subspace in the weight filtration. Theorem 3 is proved.

\section{Rational homology of schemes which are disjoint unions of varieties}

$$
\left(G^{m}\right)^{a} \times A^{b}
$$

Proof of Theorem 4. We will compute the Borel-Moore homology of $X$ using the spectral sequence associated to the filtration of $X$ by closed subspaces $X=X_{n} \supset$ $X_{n-1} \supset \cdots \supset X_{0} \supset \emptyset$, where $X_{i}$ is the union of all the pieces of dimension $\leqslant i$. In fact, this is precisely analogous to the spectral sequence used in Section 2 to compute the motivic homology of $X$.

The spectral sequence has the form

$$
E_{p q}^{1}=H_{p+q}^{B M}\left(X_{p}, X_{p-1} ; \mathbf{Z}\right) \Rightarrow H_{p+q}^{B M}(X, \mathbf{Z}) .
$$

It has homological numbering, meaning that the differential $d_{i}$ has bidegree $(-i$, $i-1)$. The $E^{1}$ term is very simple:

$$
\begin{aligned}
E_{p q}^{1} & =H_{p+q}^{B M}\left(X_{p}-X_{p-1}, \mathbf{Z}\right) \\
& =H_{p+q}^{B M}\left(\coprod T_{p}, \mathbf{Z}\right),
\end{aligned}
$$

where $T_{p}$ runs over the set of $p$-dimensional pieces of $X$,

$$
=\oplus_{T_{p}} \Lambda^{p-q} M\left(T_{p}\right) .
$$

Here, $M(T)=H^{1}(T, \mathbf{Z})$.

Example: $\operatorname{dim} X=3$.

$$
\begin{aligned}
& \oplus_{T_{0}} \mathbf{Z}_{\overleftarrow{z}} \oplus_{T_{1}} M\left(T_{1}\right) \overleftarrow{\leftarrow} \oplus_{T_{2}} \Lambda^{2} M\left(T_{2}\right) \longleftarrow \oplus_{T_{3}} \Lambda^{3} M\left(T_{3}\right) \\
& \begin{array}{r}
\oplus_{T_{1}} \mathbf{Z}_{\longleftarrow-\cdots} \oplus_{T_{2}} M\left(T_{2}\right) \longleftarrow \oplus_{T_{3}} \Lambda^{2} M\left(T_{3}\right) \\
\oplus_{T_{2}} \mathbf{Z} \longleftarrow-\oplus_{T_{3}} M\left(T_{3}\right)
\end{array} \\
& \oplus_{T_{3}} \mathbf{Z}
\end{aligned}
$$

Here, the top row has weight 0 , the next has weight -2 , the next has weight -4 , and the bottom row has weight -6 , as we will now explain for $X$ of any dimension. 
The weight filtration on $H_{*}^{B M}(X, \mathbf{Q})$ is known explicitly for $X=\left(G_{m}\right)^{a} \times$ $A^{b}$; namely, $H^{k}\left(T_{i}, \mathbf{Q}\right)$ is pure of weight $2 k$, so that Poincare duality (with the usual shift of weights, $\left.H_{k}^{B M}\left(T_{i}, \mathbf{Q}\right) \cong H^{2 i-k}\left(T_{i}, \mathbf{Q}(i)\right)\right)$ makes $H_{k}^{B M}\left(T_{i}, \mathbf{Q}\right)$ pure, of weight $2(2 i-k)-2 i=2(i-k)$. (The weight filtration is an increasing filtration $W_{*}$ on $H_{k}^{B M}(X, \mathbf{Q})$ for any complex scheme $X$. The associated graded groups are always concentrated in degrees $-k$ to 0 [6].) Since all the differentials in the spectral sequence $\otimes \mathbf{Q}$ must be strictly compatible with weights, the differentials other than $d_{1}$ are 0 after tensoring with $\mathbf{Q}$. This degeneration gives an explicit calculation of $\operatorname{gr}_{*}^{W} H_{*}^{B M}(X, \mathbf{Q})$ as the homology of the chain complexes pictured above. Theorem 4 is proved.

In particular, $W_{-2 i} H_{2 i}^{B M}(X, \mathbf{Q})=\oplus_{T_{i}} \mathbf{Q} / \oplus_{T_{i+1}}\left(M\left(T_{i+1}\right) \otimes \mathbf{Q}\right)$ is precisely what we computed $C H_{i} X$ to be, tensored with $\mathbf{Q}$, as Theorem 3 predicts.

\section{Splitting the homology of toric varieties, and its application to Chow cohomology (Theorems 5 and 6)}

One of the main open problems about the operational Chow cohomology ring $A^{*} X$ has been whether there is a natural homomorphism $A^{*} X \rightarrow H^{*}(X, \mathbf{Z})$ for all complex varieties $X$. This is true for smooth varieties, where $A^{*} X$ is the usual Chow ring of algebraic cycles, but in general the definition of $A^{*} X$ is much more abstract. In this section, we use Lieberman's trick (originally applied to abelian varieties, as in [9, p. 219]) to define a natural map $A^{*} X \otimes \mathbf{Q} \rightarrow H^{*}(X, \mathbf{Q})$ for compact toric varieties $X$. Section 7 shows that this would not be possible (with a reasonable assumption on the map) for general schemes $X$, even linear schemes. See Section 8 for further comments.

THEOREM 5. For each toric variety $X$, there is a natural grading of each group $H_{i}^{B M}(X, \mathbf{Q})$ and $H^{i}(X, \mathbf{Q})$ which splits the weight filtration and is compatible with products and mixed Hodge structures.

In fact, the argument gives a more precise statement with $\mathbf{Q}$ replaced by $\mathbf{Z}[1 / k]$ for a certain number $k=k(i)$, but I will ignore this refinement.

Proof. The idea is very simple. A toric variety $X$ is described by a fan, that is, an arrangement of cones in a lattice $\mathbf{Z}^{k}$ satisfying certain properties [11]. For each positive integer $n$, there is an endomorphism of $X$ corresponding to multiplying the lattice by $n$. This gives an action of the multiplicative monoid of positive integers, $\mathbf{N}^{>0}$, on any toric variety $X$. Considering the resulting action of $\mathbf{N}^{>0}$ on $H^{i}(X, \mathbf{Q})$, we get a natural grading of $H^{i}(X, \mathbf{Q})$,

$$
H^{i}(X, \mathbf{Q})=\oplus H_{2 j}^{i}(X, \mathbf{Q}),
$$


where $H_{2 j}^{i}$ is the subspace of $H^{i}$ on which $\mathbf{N}^{>0}$ acts by $n \mapsto n^{j}$. Similarly for Borel-Moore homology. The Chow group $C H_{i} X \otimes \mathbf{Q}$ is simply the weight $-2 i$ graded piece of $H_{2 i}^{B M}(X, \mathbf{Q})$, which gives the splitting mentioned above. In general, this grading is an explicit splitting of Deligne's weight filtration for toric varieties. From the definition, it is obvious that this extra grading on $H^{*}(X, \mathbf{Q})$ is compatible with products.

In characteristic $p>0$, the endomorphism of a toric variety corresponding to $p \in \mathbf{N}^{>0}$ is the Frobenius endomorphism. So the $\mathbf{N}^{>0}$-action on toric varieties means that they belong to the small class of varieties for which the Frobenius endomorphism lifts from characteristic $p$ to characteristic 0 , for all primes $p$.

To justify my statements about the action of $\mathbf{N}^{>0}$ on $H_{i}^{B M}(X, \mathbf{Q})$ (not every representation of the monoid $\mathbf{N}^{>0}$ is so simple), we look at the spectral sequence converging to $H_{*}^{B M}(X, \mathbf{Q})$ which was described in Section 5. This is the spectral sequence associated to the filtration of $X$ by closed subspaces $X=Y_{0} \supset Y_{1} \supset$ $\cdots \supset Y_{n} \supset \emptyset$, where $Y_{i}$ is the union of the torus orbits of dimension $\leqslant n-i$. The monoid $\mathbf{N}^{>0}$ preserves this filtration of $Y$, so it acts on the spectral sequence, and its action on the $E^{1}$ term (which is just the direct sum of the Borel-Moore homology of all the torus orbits) is very simple: $n \in \mathbf{N}^{>0}$ acts by $n^{i}$ on row $\operatorname{dim}(X)-i$.

This lets us reprove the degeneration (always $\otimes \mathbf{Q}$ ) of this spectral sequence in the case of toric varieties: the differential $d_{i}$ moves $i-1$ rows up, so by $\mathbf{N}^{>0}$-equivariance of the differentials they are all 0 except for $d_{1}$. Moreover, the filtration on $H_{i}^{B M}(X, \mathbf{Q})$ coming from the spectral sequence has a unique $\mathbf{N}^{>0}$ equivariant splitting, since the different groups contributing to $H_{i}^{B M}(X, \mathbf{Q})$ are all of different weights with respect to $\mathbf{N}^{>0}$, and any extension of representations of $\mathbf{N}^{>0}$ over $\mathbf{Q}$ of different weights has a unique splitting. This is an elementary argument which we omit, the same as that used to define the Adams grading on $K$-theory tensored with Q; see [1, p. 140], for example.

Finally, from the geometric origin of this splitting of $H_{i}^{B M}(X, \mathbf{Q})$, we see that it gives a splitting of the mixed Hodge structure $H_{i}^{B M}(X, \mathbf{Q})$ as a direct sum of pure Hodge structures of different weights. The weight $-2 j$ part of $H_{i}^{B M}(X, \mathbf{Q})$ is a direct sum of several copies of the Tate Hodge structure $\mathbf{Q}(j)$. Theorem 5 is proved.

Proof of Theorem 6. Let $X$ be a compact toric variety over $\mathbf{C}$. Since $C H_{i} X \otimes \mathbf{Q} \cong$ $W_{-2 i} H_{2 i}^{B M}(X, \mathbf{Q})$ (Theorem 3) and $A^{i} X \cong \operatorname{Hom}\left(C H_{i} X, \mathbf{Z}\right)$ (Theorem 2), we have a natural isomorphism $A^{i} X \otimes \mathbf{Q}=\operatorname{gr}_{2 i}^{W} H^{2 i}(X, \mathbf{Q})$. Since Theorem 5 gives an explicit splitting of the weight filtration on $H^{2 i}(X, \mathbf{Q})$, it gives a homomorphism $A^{i} X \otimes \mathbf{Q} \hookrightarrow H^{2 i}(X, \mathbf{Q})$. In fact, we can describe the image just in terms of the mixed Hodge structure on $H^{*}(X, \mathbf{Q})$. Theorem 5 says that the mixed Hodge 
structure on $H^{k}(X, \mathbf{Q})$ is a direct sum of pieces isomorphic to sums of the $\mathbf{Q}(j)$ for various $j$. Whenever one has a mixed Hodge structure of this form, its splitting into pure-weight pieces is unique and is given by

$$
\operatorname{gr}_{2 j}^{W} H^{k}(X, \mathbf{Q})=W_{2 j} H^{k}(X, \mathbf{Q}) \cap F^{j} H^{k}(X, \mathbf{C}) \subset H^{k}(X, \mathbf{Q}) .
$$

It follows that this must be the splitting given by Theorem 5. In particular, the image of the homomorphism $A^{i} X \otimes \mathbf{Q} \hookrightarrow H^{2 i}(X, \mathbf{Q})$ defined above is exactly

$$
W_{2 i} H^{2 i}(X, \mathbf{Q}) \cap F^{i} H^{2 i}(X, \mathbf{C}),
$$

which is just $H^{2 i}(X, \mathbf{Q}) \cap F^{i} H^{2 i}(X, \mathbf{C})$ for $X$ compact.

\section{An example}

We define a normal projective surface $Y$ with one of the simplest nonrational singularities, obtained by blowing down a nodal rational curve. $Y$ is in fact a linear variety. But the mixed Hodge structure on $H^{2}(Y, \mathbf{Q})$ is a nontrivial extension of a sum of the $\mathbf{Q}(-1)$ by $\mathbf{Q}(0)$, and the Bloch-Gillet-Soulé map

$$
A^{1} Y \otimes \mathbf{Q} \rightarrow \operatorname{gr}_{2}^{W} H^{2}(Y, \mathbf{Q}) \cap F^{1} \operatorname{gr}_{2}^{W} H^{2}(Y, \mathbf{C})
$$

does not lift to $H^{2}(Y, \mathbf{Q}) \cap F^{1} H^{2}(Y, \mathbf{C})$. By considering a family of varieties containing $Y$, we show that there is no map $A^{1} X \rightarrow H^{2}(X, \mathbf{Q})$ with good properties for general varieties $X$.

We now describe the example: it is probably the simplest surface with the singularity given by blowing down a nodal rational curve to a point. We consider a nodal cubic curve $C$ in $\mathbf{P}^{2}$, and we blow up $\geqslant 10$ points in $\mathbf{P}^{2}$ to make the proper transform $C^{\prime}$ of $C$ have negative self-intersection number, so that we can hope to blow $C^{\prime}$ down to a point. In order to make sure that the blown-down surface is projective, we have to choose carefully which points on $C$ to blow up.

Namely, let $D$ be a curve of any degree $d \geqslant 4$ in $\mathbf{P}^{2}$ which intersects $C$ transversely, at smooth points of $C$. Let $X$ be the surface obtained by blowing $\mathbf{P}^{2}$ up at the $3 d$ points of $C \cap D$. Let $C^{\prime}$ and $D^{\prime}$ be the proper transforms of $C$ and $D$. Their self-intersection numbers are given by

$$
\begin{aligned}
& \left(C^{\prime}\right)^{2}=9-3 d<0, \\
& \left(D^{\prime}\right)^{2}=d^{2}-3 d>0 .
\end{aligned}
$$

Moreover, $C^{\prime}$ and $D^{\prime}$ are disjoint in $X$. So we can blow down the nodal rational curve $C^{\prime}$ to get a singular projective surface $Y\left(D^{\prime}\right.$ gives a line bundle whose multiples have enough sections on $Y$ to show that $Y$ is projective). 
Explicitly, if $d=4$, then $Y$ imbeds as the singular quartic surface

$$
w\left(y^{2} z-x^{2} z-x^{3}\right)+f(x, y, z)=0,
$$

in $\mathbf{P}^{3}$, where $y^{2} z-x^{2} z-x^{3}=0$ is the equation of the nodal cubic $C$ and $f$ is the equation of $D \subset \mathbf{P}^{2}$. In this case, where $d=4, Y$ arises naturally in the study of degenerations of $\mathrm{K} 3$ surfaces. For any $d, Y$ is obtained by blowing down an anticanonical curve in the rational surface $X$, and $Y$ is Gorenstein with $K_{Y}=0$, but for $d \geqslant 5$ the singular point of $Y$ is not a hypersurface singularity. We will not need these observations, however.

The cohomology of $Y$ is computed by the following exact sequence.

$$
\begin{aligned}
& 0 \rightarrow H^{1}\left(C^{\prime}, \mathbf{Z}\right) \quad \rightarrow \quad H^{2}(Y, \mathbf{Z}) \quad \rightarrow \quad H^{2}(X, \mathbf{Z}) \quad \rightarrow \quad H^{2}\left(C^{\prime}, \mathbf{Z}\right) \\
& =\mathbf{Z} \quad=\mathbf{Z}^{3 d+1} \quad=\mathbf{Z}
\end{aligned}
$$

Consider the line bundle $L$ on the smooth surface $X$ given by the difference of two of the $3 d$ exceptional divisors. Then $[L] \in H^{2}(X, \mathbf{Z})$ restricts to 0 in $H^{2}\left(C^{\prime}\right.$, $\mathbf{Z}$ ), but $L$ itself is nontrivial on $C^{\prime}$ : it corresponds to the difference of two smooth points on $C^{\prime}$, which gives a nontrivial element of

$$
\operatorname{Pic}^{0}\left(C^{\prime}\right)=\mathbf{C}^{*} \text {. }
$$

In fact, by choosing the curve $D$ generically, we can arrange that $L$ will restrict to an element of $\mathbf{C}^{*}$ which is not a root of unity. Then $L$ restricts to a nonzero element of Pic $C \otimes \mathbf{Q}$, and so $[L] \in \operatorname{Pic} X \otimes \mathbf{Q}=H^{2}(X, \mathbf{Q})$ is not in the image of Pic $Y \otimes \mathbf{Q}$, though it is in the image of $H^{2}(Y, \mathbf{Q})$.

Thus $Y$ does not have as many line bundles as one might think. To show that this corresponds to the nonsplitting of the mixed Hodge structure on $H^{2}(Y, \mathbf{Q})$, we will prove that $Y$ satisfies the singular Hodge conjecture for line bundles:

\section{PROPOSITION 3.}

$$
\operatorname{im}\left(\operatorname{Pic} Y \rightarrow H^{2}(Y, \mathbf{Z})\right)=\left\{x \in H^{2}(Y, \mathbf{Z}): x_{\mathbf{C}} \in F^{1} H^{2}(Y, \mathbf{C})\right\} .
$$

This is false for some varieties $Y$, in fact for the variety $Y$ obtained by exactly the construction above with a cuspidal cubic instead of a nodal cubic (in that case $H^{1}\left(C^{\prime}, \mathbf{Z}\right)=0$, so the mixed Hodge structure on $H^{2}(Y, \mathbf{Q})$ is trivial, but still, since $\mathrm{Pic}^{0} \mathrm{C}^{\prime}=\mathbf{C} \neq 0$, the argument above shows that not all of $H^{2}(Y, \mathbf{Q})$ comes from line bundles). This counterexample to the singular Hodge conjecture for line bundles was found by Barbieri-Viale and Srinivas [2].

Proof of Proposition 3. We have to prove that the singular Hodge conjecture is true in our case, where $C^{\prime}$ is a nodal cubic. By the exponential sequence, we need to show that $F^{1} H^{2}(Y, \mathbf{C})$ maps to 0 in $H^{2}(Y, O)$. 
We begin by computing $H^{2}(Y, O)$ to see that it is not too big. (Namely, it is $\mathbf{C}$.) Look at the exact sequence on the smooth surface $X$,

$$
0 \rightarrow O_{X}\left(-C^{\prime}\right) \rightarrow O_{X} \rightarrow O_{C^{\prime}} \rightarrow 0 .
$$

Since $H^{i}(X, O)=0$ for $i>0$, we have that

$$
\mathbf{C}=H^{1}(S, O) \stackrel{\cong}{\rightarrow} H^{2}(X, O(-S))=H^{2}\left(Y, R f_{*} O(-S)\right) .
$$

Here, $f: X \rightarrow Y$ is the contraction map. The point is that $R f_{*} O(-S)$ is directly related to $O_{Y}$. In fact, $f_{*} O(-S)$ is the ideal sheaf $I_{p} \subset O_{Y}$ of the singular point $p \in Y$, and the higher cohomology sheaves $R^{i} f_{*} O(-S)$ are 0 for $i>0$. To prove the latter fact, notice that the canonical bundle $K_{S}$ of the nodal curve is trivial ( $S$ is a degenerate elliptic curve), which means that

$$
\left.\left(K_{X}+S\right)\right|_{S}=0 \in \operatorname{Pic}(S) .
$$

But the Kawamata-Viehweg vanishing theorem implies that for a projective birational morphism $f: X \rightarrow Y$ with $X$ smooth, if $L$ is a line bundle on $X$ with $\left.\operatorname{deg}(L)\right|_{C} \geqslant 0$ for all curves $C$ on $X$ with $f(C)=$ point, then $R^{i} f_{*}\left(K_{X}+L\right)=0$ for $i>0$ [8]. It follows that $R^{i} f_{*} O(-S)=0$ for $i>0$ as claimed: $R f_{*} O(-S)$ is just the ideal sheaf $I_{p}$. So

$$
H^{2}(Y, O)=H^{2}\left(Y, I_{p}\right)=H^{2}\left(Y, R f_{*} O(-S)\right),
$$

where we computed the latter group to be $H^{1}(S, O) \cong \mathbf{C}$.

Having computed that $H^{2}(Y, O)=\mathbf{C}$, we next need to show that

$$
\operatorname{ker}\left(H^{i}(Y, \mathbf{C}) \rightarrow H^{i}(Y, O)\right) \subset F^{1} H^{i}(Y, \mathbf{C})
$$

for any $i$, which we will actually prove for an arbitrary compact variety $Y$. This is easy once one understands Deligne's definition of the Hodge filtration $F^{*}$ on $H^{i}(Y, \mathbf{C})$. Namely, one chooses a map $\left(Y_{n}\right) \rightarrow Y$ from a simplicial scheme $\left(Y_{n}\right)$ to $Y$ such that the varieties $Y_{n}$ are smooth and compact, and all the face maps as well as the map $Y_{0} \rightarrow Y$ are proper and surjective. This rather weak assumption turns out to imply that the map $H^{*}(Y, \mathbf{Z}) \rightarrow H^{*}\left(\left(Y_{n}\right), \mathbf{Z}\right)$ (mapping to the cohomology of the constant sheaf $\mathbf{Z}$ on the simplicial scheme $\left.\left(Y_{n}\right)\right)$ is an isomorphism. Since the varieties $Y_{n}$ are smooth and compact, we can compute at least their cohomology with $\mathbf{C}$ coefficients using de Rham cohomology, so that

$$
H^{*}(Y, \mathbf{C})=H^{*}\left(\left(Y_{n}\right), \Omega^{*}\right)
$$

The Hodge filtration on $H^{*}(Y, \mathbf{C})$ comes from the obvious filtration of the de Rham complex with $F^{i}$ corresponding to $\Omega^{j}$ for $j \geqslant i$. In the case we want, this means that

$$
F^{1} H^{*}(Y, \mathbf{C})=\operatorname{ker}\left(H^{*}\left(\left(Y_{n}\right), \Omega^{*}\right) \rightarrow H^{*}\left(\left(Y_{n}\right), O\right)\right)
$$


But the map from $H^{*}(Y, \mathbf{C})$ to $H^{*}\left(\left(Y_{n}\right), O\right)$ factors through $H^{*}(Y, O)$. It follows that

$$
\operatorname{ker}\left(H^{2}(Y, \mathbf{C}) \rightarrow H^{2}(Y, O)\right) \subset F^{1} H^{2}(Y, \mathbf{C}),
$$

as we want.

But, in the case at hand, $\operatorname{ker}\left(H^{2}(Y, \mathbf{C}) \rightarrow H^{2}(Y, O)\right)$ is a subspace of codimension $\leqslant 1$ in $H^{2}(Y, \mathbf{C})$, while $F^{1} H^{2}(Y, \mathbf{C})$ has codimension 1 by the exact sequence

$$
0 \rightarrow H^{1}\left(C^{\prime}, \mathbf{C}\right) \rightarrow H^{2}(Y, \mathbf{C}) \rightarrow H^{2}(X, \mathbf{C}) \rightarrow H^{2}\left(C^{\prime}, \mathbf{C}\right),
$$

which is strictly compatible with the Hodge filtration. (The nodal cubic $C^{\prime}$ has $H^{1}\left(C^{\prime}, \mathbf{C}\right) \cong \mathbf{C}$ all in $F^{0}$, with $F^{1}=0$, while the blow-up $X$ of $\mathbf{P}^{2}$ has $H^{2}(X, \mathbf{C})$ all in $F^{1}$.) It follows that

$$
\operatorname{ker}\left(H^{2}(Y, \mathbf{C}) \rightarrow H^{2}(Y, O)\right)=F^{1} H^{2}(Y, \mathbf{C}),
$$

which proves the singular Hodge conjecture for line bundles on $Y$. Proposition 3 is proved.

Now our earlier analysis of the line bundles on $Y$ gives information about the mixed Hodge structure on $H^{2} Y$, thanks to Proposition 3. We showed that

$$
\operatorname{im}\left(\operatorname{Pic} Y \otimes \mathbf{Q} \rightarrow \operatorname{gr}_{2}^{W} H^{2}(Y, \mathbf{Q})\right) \varsubsetneqq \operatorname{gr}_{2}^{W} H^{2}(Y, \mathbf{Q}) \cap F^{1} \operatorname{gr}_{2}^{W} H^{2}(Y, \mathbf{C}) .
$$

It now follows that the map

$$
H^{2}(Y, \mathbf{Q}) \cap F^{1} H^{2}(Y, \mathbf{C}) \rightarrow \operatorname{gr}_{2}^{W} H^{2}(Y, \mathbf{Q}) \cap F^{1} \operatorname{gr}_{2}^{W} H^{2}(Y, \mathbf{C})
$$

is not surjective. This means that the mixed Hodge structure on $H^{2}(Y, \mathbf{Q})$ is a nontrivial extension of $\mathbf{Q}(-1)^{3 d}$ by $\mathbf{Q}(0)$. (The convention is that the mixed Hodge structure $\mathbf{Q}(j)$ has weight $-2 j$.)

Related to this, we can draw a conclusion about the Chow cohomology group $A^{1} Y$. The theory $A^{1}$ does not see the line bundles $\mathbf{C}^{*} \subset$ Pic $\left(C^{\prime}\right)$. In fact, Kimura's computation of Chow cohomology [19] implies that $A^{*}$ of a singular variety injects into $A^{*}$ of a smooth resolution (which is the usual Chow ring of a smooth variety) with an explicit cokernel. As a result, for the nodal cubic $C^{\prime}$ (with resolution $\left.\mathbf{P}^{1}\right)$, one checks that $A^{1} C^{\prime}=H^{2}\left(C^{\prime}, \mathbf{Z}\right)=\mathbf{Z}$. Also, $A^{1} X=H^{2}(X$, Z). Kimura's theorem applied to $Y$ then says that

$$
\begin{aligned}
A^{1} Y & =\operatorname{ker}\left(A^{1} X \rightarrow A^{1} C^{\prime}\right) \\
& =\operatorname{ker}\left(H^{2}(X, \mathbf{Z}) \rightarrow H^{2}\left(C^{\prime}, \mathbf{Z}\right)\right) \\
& =\operatorname{im}\left(H^{2}(Y, \mathbf{Z}) \rightarrow H^{2}(X, \mathbf{Z})\right)
\end{aligned}
$$


That is,

$$
A^{1} Y \otimes \mathbf{Q}=\operatorname{gr}_{2}^{W} H^{2}(Y, \mathbf{Q}),
$$

which is all in $F^{1}$, and the previous paragraph then shows that the map $A^{1} Y \otimes \mathbf{Q} \rightarrow$ $\mathrm{gr}_{2}^{W} H^{2}(Y, \mathbf{Q})$ does not lift to

$$
H^{2}(Y, \mathbf{Q}) \cap F^{1} H^{2}(Y, \mathbf{C}) .
$$

THEOREM 7. There is no functorial homomorphism $A^{1} X \otimes \mathbf{Q} \rightarrow H^{2}(X, \mathbf{Q})$ for general complex varieties $X$ (or even for normal complex projective linear varieties $X)$ which agrees with the obvious map for $X$ smooth and which is well behaved in families in the following sense. If $\left\{Y_{b}\right\}$ is a family of varieties over a base variety $B$ such that the sheaf $H^{2}\left(Y_{b}, \mathbf{Q}\right), b \in B$, is locally constant, and if $x_{b} \in H^{2}\left(Y_{b}, \mathbf{Q}\right)$ is a section of this sheaf over an analytic open set $U \subset B$, then the set of $b \in U$ such that $x_{b}$ is in the image of the supposed map $A^{1} Y_{b} \otimes \mathbf{Q} \rightarrow H^{2}\left(Y_{b}\right.$, Q) is a countable union of analytic constructible subsets of $U$.

An analytic constructible subset means a finite union of differences between closed analytic subsets. This property seems to be a reasonable thing to ask of a map $A^{*} X \otimes \mathbf{Q} \rightarrow H^{*}(X, \mathbf{Q})$. The analogous property is true for the Chern character $K_{0} X \otimes \mathbf{Q} \rightarrow H^{*}(X, \mathbf{Q})$ and for the Bloch-Gillet-Soulé map $A^{i} X \otimes \mathbf{Q} \rightarrow$ $\mathrm{gr}_{2}^{W} H^{2 i}(X, \mathbf{Q})$.

Proof of Theorem 7. The variety $Y$ defined above depends on a choice of a curve of degree $d \geqslant 4$ in $\mathbf{P}^{2}$. Fix $d \geqslant 4$, and let $B_{0}$ be the space of curves of degree $d$ in $\mathbf{P}^{2}$ which meet the nodal cubic $C \subset \mathbf{P}^{2}$ transversely at smooth points of $C$. Then the construction of this section gives a family of varieties $\left\{Y_{b}\right\}$ parameterized by $b \in B_{0}$. Let $B$ be a connected component of the covering space of $B_{0}$ with fiber the symmetric group $S_{3 d}$, so that $B$ parameterizes curves of degree $D$ as above together with an ordering of the $3 d$ intersection points of the curve with the nodal cubic $C$. The pulled-back family of varieties $\left\{Y_{b}\right\}$ over $B$ has the advantage that there is a natural element $E_{1}-E_{2} \in A^{1} Y_{b}$ for all $b \in B$, corresponding to the divisor $E_{1}-E_{2}$ on the smooth resolution $X_{b}$ of $Y_{b}$, as we found earlier in this section.

Suppose that there is a functorial homomorphism $A^{1} X \otimes \mathbf{Q} \rightarrow H^{2}(X, \mathbf{Q})$ as in the theorem. Since it agrees with the obvious map for $X$ smooth, the composition

$$
A^{1} X \otimes \mathbf{Q} \rightarrow H^{2}(X, \mathbf{Q}) \rightarrow \operatorname{gr}_{2}^{W} H^{2}(X, \mathbf{Q})
$$

must be the Bloch-Gillet-Soulé map (since, say for $X$ compact, $\operatorname{gr}_{2}^{W} H^{2}(X, \mathbf{Q})$ is the image of $H^{2}(X, \mathbf{Q})$ in the cohomology of a smooth resolution of $\left.X\right)$. 
Since the homomorphism $A^{1} Y_{b} \otimes \mathbf{Q} \rightarrow \operatorname{gr}_{2}^{W} H^{2}\left(Y_{b}, \mathbf{Q}\right)$ which really exists is an isomorphism for all $b \in B$, the subspaces $V_{b}:=\operatorname{im}\left(A^{1} Y_{b} \otimes \mathbf{Q} \rightarrow H^{2}\left(Y_{b}\right.\right.$, Q)) have constant dimension (equal to $3 d$, in fact), and $V_{b}$ is a splitting of the weight filtration on the vector space $H^{2}\left(Y_{b}, \mathbf{Q}\right)$. So our assumption on the homomorphism $A^{1} X \rightarrow H^{2}(X, \mathbf{Q})$ implies that, over each open ball $U \subset B$, there is one flat section $x_{b} \in H^{2}\left(Y_{b}, \mathbf{Q}\right)$ which is the image of $E_{1}-E_{2} \in A^{1}\left(Y_{b}\right) \otimes \mathbf{Q}$ for all $b \in U$ outside a countable union of lower-dimensional analytic subspaces. By patching together these sections over different open balls of $B$, we find a global flat section $x_{b} \in H^{2}\left(Y_{b}, \mathbf{Q}\right), b \in B$, which lifts the flat section $E_{1}-E_{2}$ of $\mathrm{gr}_{2}^{W}\left(Y_{b}, \mathbf{Q}\right)$.

The point is that there are special points $b \in B$ for which $E_{1}-E_{2} \in \mathrm{gr}_{2}^{W} H^{2}\left(Y_{b}\right.$, Q) lies in the image of Pic $Y_{b} \otimes \mathbf{Q} \rightarrow H^{2}\left(Y_{b}, \mathbf{Q}\right) \rightarrow \operatorname{gr}_{2}^{W} H^{2}\left(Y_{b}, \mathbf{Q}\right)$ : this happens whenever the difference of the first two points of $C \cap D$ represents a root of unity in $\operatorname{Pic}^{0}(C)=\mathbf{C}^{*}$. (Recall that $C$ is a fixed nodal cubic curve in $\mathbf{P}^{2}$, and that a point of $B$ determines a degree- $d$ curve $D$ together with an ordering of the $3 d$ points of $C \cap D$.) Pick such a special point $b$, and observe that the global flat section $x_{b} \in H^{2}\left(Y_{b}, \mathbf{Q}\right)$ differs at this point from the image of an element of Pic $Y_{b} \otimes \mathbf{Q}$, thus from an element of $H^{2}\left(Y_{b}, \mathbf{Q}\right) \cap F^{1} H^{2}\left(Y_{b}, \mathbf{C}\right)$, by an element of $H^{1}\left(C_{b}\right.$, $\mathbf{Q})=\mathbf{Q} \subset H^{2}\left(Y_{b}, \mathbf{Q}\right)$. (See the earlier calculation of $H^{2}\left(Y_{b}, \mathbf{Q}\right)$.) Since the sheaf $H^{1}\left(C_{b}, \mathbf{Q}\right)$ is isomorphic to the constant sheaf $\mathbf{Q}$ over $B$, we can modify $x_{b}$ to produce a global flat section $y_{b} \in H^{2}\left(Y_{b}, \mathbf{Q}\right)$ over $b \in B$ which lifts $E_{1}-E_{2} \in$ $\mathrm{gr}_{2}^{W} H^{2}\left(Y_{b}, \mathbf{Q}\right)$ and which belongs to $F^{1} H^{2}\left(Y_{b}, \mathbf{C}\right)$ at one point of $B$. But a flat section of a variation of mixed Hodge structures over an algebraic base $B$ which is in $F^{i}$ at one point belongs to $F^{i}$ at every point, by Steenbrink and Zucker [25, p. 517]. So the classes $y_{b} \in H^{2}\left(Y_{b}, \mathbf{Q}\right)$ belong to $F^{1} H^{2}\left(Y_{b}, \mathbf{C}\right)$ for all $b \in B$. Our previous proposition shows, however, that no lift of $E_{1}-E_{2} \in \operatorname{gr}_{2}^{W} H^{2}\left(Y_{b}, \mathbf{Q}\right)$ can belong to $F^{1} H^{2}\left(Y_{b}, \mathbf{C}\right)$ when the difference of the first two points of $C \cap D$ is not a root of unity in $\operatorname{Pic}^{0}(C) \cong \mathbf{C}^{*}$. We have a contradiction.

Thus there is no map $A^{1} X \otimes \mathbf{Q} \rightarrow H^{2}(X, \mathbf{Q})$ with the required properties. Theorem 7 is proved.

\section{Problems on Chow cohomology}

We begin by describing some general questions about operational Chow cohomology $A^{*} X$, mostly for compact complex varieties. Note that Voevodsky's motivic cohomology ring $H_{M}^{a}(X, \mathbf{Z}(b))$ is probably more useful than $A^{*} X$ for singular schemes $X[26]$.

Operational Chow cohomology is defined to be, in a sense, the weakest of all possible Chow cohomology theories: any reasonable Chow cohomology theory will act on Chow (homology) groups, and so will map to $A^{*} X$. For example, there 
is a natural map from the Adams-graded pieces of algebraic $K$-theory, gr* $K_{0} X \otimes$ $\mathbf{Q}$, to $A^{*} X \otimes \mathbf{Q}$. As a result, it is not easy to define maps from $A^{*} X$ to any other sort of cohomology theory. In particular, the question of the existence of a natural homomorphism $A^{*} X \rightarrow H^{*}(X, \mathbf{Z})$ remained open until this paper.

Bloch, Gillet, and Soulé [5] showed that there is at least a natural map from $A^{i} X \otimes \mathbf{Q}$ to $\operatorname{gr}_{2 i}^{W} H^{2 i}(X, \mathbf{Q})$, which is a quotient of $H^{2 i}(X, \mathbf{Q})$ for $X$ compact. If $X$ is a compact toric variety, we showed in Theorem 6 that this homomorphism lifts uniquely to a homomorphism $A^{i} X \otimes \mathbf{Q} \rightarrow H^{2 i}(X, \mathbf{Q}) \cap F^{i} H^{2 i}(X, \mathbf{C})$ (which is in fact an isomorphism). And of course there is a homomorphism $A^{i} X \otimes \mathbf{Q} \rightarrow$ $H^{2 i}(X, \mathbf{Q}) \cap F^{i} H^{2 i}(X, \mathbf{C})$ for all smooth varieties $X$, since $A^{i} X$ coincides with the Chow group $C H_{n-i} X$ of codimension- $i$ algebraic cycles for $X$ smooth. So the positive results on this question for toric varieties should extend to all varieties whose singularities are not too bad. Specifically, Fulton asks if there is a natural homomorphism

$$
A^{i} X \otimes \mathbf{Q} \rightarrow H^{2 i}(X, \mathbf{Q}) \cap F^{i} H^{2 i}(X, \mathbf{C})
$$

for all varieties $X$ with rational singularities. This is true for $i=1$, since Kollár and Mori [20] have proved that $\operatorname{Pic} X \otimes \mathbf{Q} \cong A^{1} X \otimes \mathbf{Q}$ if $X$ has rational singularities, and Pic $X$ obviously maps into $H^{2}(X, \mathbf{Z}) \cap F^{1} H^{2}(X, \mathbf{C})$.

The normal surface in Section 7, with one of the simplest nonrational singularities, shows that there is no map $A^{1} X \otimes \mathbf{Q} \rightarrow H^{2}(X, \mathbf{Q})$ with good properties for general varieties $X$.

One can also ask for integral versions of these results. For toric varieties, and more generally for spherical varieties, the map Pic $X \rightarrow A^{1} X$ is an isomorphism (Brion), and so there is a natural homomorphism $A^{1} X \rightarrow H^{2}(X, \mathbf{Z})$. Both statements fail if $X$ only has rational singularities, as Alessio Corti found, but one may expect that there is a finer class of singularities including the toric ones which would imply these statements. It is tempting to guess more generally that there is a natural homomorphism $A^{i} X \rightarrow H^{2 i}(X, \mathbf{Z})$ for toric varieties at least.

In the same spirit, one can ask for an integral version of Theorem 3. Namely, is the homomorphism $C H_{i} X \rightarrow H_{2 i}^{B M}(X, \mathbf{Z})$ a split injection of abelian groups, say for toric varieties $X$ ? The answer is yes for smooth (but not necessarily compact) toric varieties, by Franz [10, Corollary 1.3].

\section{Acknowledgements}

This work was supported by a Sloan Foundation Fellowship and by NSF grant DMS-1303105.

I am indebted to Fulton's questions about these matters. I thank Alessio Corti, Rob Morelli, Masaki Hanamura, and Frank Sottile for useful conversations; Corti 
showed me how to use the Kawamata-Viehweg vanishing theorem as in Section 7. Angelo Vistoli found Theorem 4 independently.

\section{References}

[1] M. Atiyah, K-Theory (W.A. Benjamin, 1967).

[2] L. Barbieri Viale and V. Srinivas, 'The Néron-Severi group and the mixed Hodge structure on $H^{2}$, J. Reine Angew. Math. 450 (1994), 37-42.

[3] S. Bloch, 'Algebraic cycles and higher K-theory', Adv. Math. 61 (1986), 267-304.

[4] S. Bloch, 'The moving lemma for higher Chow groups', J. Algebraic Geom. 3 (1994), 537-568.

[5] S. Bloch, H. Gillet and C. Soulé, 'Non-archimedean Arakelov theory', J. Algebraic Geom. 4 (1995), 427-485.

[6] P. Deligne, 'Poids dans la cohomologie des variétés algébriques', in Proceedings of the International Congress of Mathematicians (Vancouver, 1974), Canad. Math. Congress, vol. 1 (1975), 79-85.

[7] D. Dugger and D. Isaksen, 'Motivic cell structures', Algebr. Geom. Topol. 5 (2005), 615-652.

[8] H. Esnault and E. Viehweg, Lectures on Vanishing Theorems (Birkhäuser, 1992).

[9] G. Faltings and C.-L. Chai, Degeneration of Abelian Varieties (Springer, 1990).

[10] M. Franz, 'The integral cohomology of toric manifolds', Tr. Mat. Inst. Steklova 252 (2006), 61-70. English translation in Proc. Steklov Inst. Math. 2006, no. 1 (252), 53-62.

[11] W. Fulton, Introduction to Toric Varieties (Princeton, 1993).

[12] W. Fulton and R. MacPherson, 'Categorical framework for the study of singular spaces', Mem. Amer. Math. Soc. 243 (1981).

[13] W. Fulton, R. MacPherson, F. Sottile and B. Sturmfels, 'Intersection theory on spherical varieties', J. Algebraic Geom. 4 (1995), 181-193.

[14] A. Huber and B. Kahn, 'The slice filtration and mixed Tate motives', Compos. Math. 142 (2006), 907-936.

[15] U. Jannsen, 'Deligne homology, Hodge D-conjecture, and motives', in Beilinson's Conjectures on Special Values of L-Functions (eds M. Rapoport, N. Schappacher and P. Schneider), (Academic Press, 1988), 305-372.

[16] U. Jannsen, Mixed Motives and Algebraic K-Theory, Lecture Notes in Mathematics, vol. 1400 (Springer, 1990).

[17] R. Joshua, 'Algebraic $K$-theory and higher Chow groups of linear varieties', Math. Proc. Cambridge Philos. Soc. 130 (2001), 37-60.

[18] S. Kelly, 'Triangulated categories of motives in positive characteristic'. arXiv:1305.5349.

[19] S. Kimura, 'Fractional intersection and bivariant theory', Comm. Algebra 20 (1992), 285-302.

[20] J. Kollár and S. Mori, 'Classification of three-dimensional flips', J. Amer. Math. Soc. 5 (1992), 533-703.

[21] M. Levine, 'Techniques of localization in the theory of algebraic cycles', J. Algebraic Geom. 10 (2001), 299-363.

[22] C. Mazza, V. Voevodsky and C. Weibel, Lecture Notes on Motivic Cohomology (Amer. Math. Soc., 2006).

[23] S. Payne, 'Equivariant Chow cohomology of toric varieties', Math. Res. Lett. 13 (2006), 29-41.

[24] M. Rosenlicht, 'Questions of rationality for solvable algebraic groups over nonperfect fields', Annali di Mat. 61 (1963), 97-120. 
[25] J. Steenbrink and S. Zucker, 'Variations of mixed Hodge structure I', Invent. Math. 80 (1985), 489-542.

[26] V. Voevodsky, 'Triangulated categories of motives over a field', in Cycles, Transfers, and Motivic Homology Theories (Princeton, 2000), 188-238.

[27] V. Voevodsky, 'Motivic cohomology groups are isomorphic to higher Chow groups in any characteristic', Int. Math. Res. Notices (7) (2002). 